EDITORIAL

\title{
Paediatric Virology: A new paediatric subspecialty? A proposal at the Workshop on Paediatric Virology, Athens, October 10, 2015
}

\author{
IOANNIS N. MAMMAS ${ }^{1}$, ANNE GREENOUGH ${ }^{2}$, MARIA THEODORIDOU $^{3}$ and DEMETRIOS A. SPANDIDOS ${ }^{1}$ \\ ${ }^{1}$ Department of Clinical Virology, University of Crete School of Medicine, Heraklion 71003, Greece; \\ ${ }^{2}$ Division of Asthma, Allergy and Lung Biology, King's College London, London SE5 9RS, UK; \\ ${ }^{3}$ First Department of Paediatrics, 'Aghia Sophia' Children's Hospital, \\ University of Athens School of Medicine, Athens 115 27, Greece
}

Received October 12, 2015; Accepted November 24, 2015

DOI: 10.3892/etm.2015.2889

Paediatric subspecialty education involves the state-ofthe-art training and practice on specific scientific fields of Paediatrics requiring clinical expertising and academic excellence (1). The medical school settings and the tertiary hospitals have been specified as the main employment sites of paediatric subspecialists, where their major professional activities include direct paediatric patient care, teaching, as well as management duties. A combination of enhanced paediatric demands and expectations have contributed to the need for standardised and comprehensive training programmes, focusing on the suitable education of paediatric subspecialty trainees.

The paediatric infectious diseases subspeciality is one of the first subspecialities, which has been included in the paediatric training programmes in several countries, worldwide (2). To date, paediatric infectious diseases professionals have achieved a distinct role within the scientific field of Paediatrics and their contribution to tertiary paediatric care is considered invaluable $(3,4)$. However, over the past decade, scientific advances on the field of Clinical Virology and Molecular Medicine have led to changes in current clinical practice as regards the management and treatment of neonates and children with viral infections. New viral infections are constantly emerging, requiring new prevention strategies and therapeutic protocols. Paediatric transplant infectious diseases are another challenge within paediatric infectious diseases, reflecting the complexity of this patient population (5). Moreover, new clinical practice guidelines for the vaccination of immunocompromised children have been established and

Correspondence to: Professor Demetrios A. Spandidos, Department of Clinical Virology, School of Medicine, University of Crete, Heraklion 71003, Greece

E-mail: spandidos@spandidos.gr

Key words: Paediatric Virology, viral infections, training, education thus regular reviewing of this matter is required (6). Additional scientific fields, including Evidence-based Medicine, Clinical Governance and Pharmacokinetic/Pharmacodynamics (7) have also a significant supportive input. These advances have highlighted the role of Paediatric Virology as a new paediatric subspeciality candidate, a new unique challenging scientific area, where paediatric professionals of the 21 st century require an advanced state-of-the-art education. The new challenges related to paediatric viral infections include emerging infections, opportunistic infections in immunocompromised patients, antimicrobial resistance and the development of new diagnostic tests, vaccines and antiviral agents, ensuring the future of this paediatric subspecialty.

The word 'Paediatric', referring to the branch of Medicine that deals with the medical care of children, derives from two ancient Greek words: ' $\pi \alpha \hat{\varsigma} \varsigma$ ' (pais), which in modern Greek has changed to ' $\pi \alpha \iota \delta$ ' (paidi), meaning the 'child' and

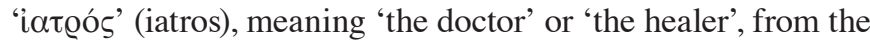
verb 'ióo $\mu \alpha \iota / \imath \hat{\omega} \mu \alpha \iota$ ' (iaome/iome), meaning 'healing'. On the other hand, the word 'Virology', the study of viruses, comes from the Latin word 'virus', meaning 'the poison', which has been in common use in the English language for many centuries and the ancient Greek word ' $\lambda$ ó $\gamma \circ \varsigma^{\prime}$ ' (logos) from the verb ' $\lambda \varepsilon{ }^{\prime} \gamma \omega$ ', meaning 'talk about'. The ancient Greek origin of virus, 'ió $\varsigma$ ', comes from the verb 'í $\eta \mu$ ' (iimi), meaning 'actuating, causing movement', such as throwing an arrow or a poison. Both words in the term 'Paediatric Virology' contain the 9th letter of the Greek alphabet ' $l$ ', the vertical line presented as an upright rod, an Homer's symbol of the strength of life. Of note, both actions of 'î $\omega \alpha$ ' supporting life and 'í $\eta \mu$ ' causing movement denote the strength and power that a children's healer needs in order to fight the small viral agents that pose a threat to the health and the life of children.

Paediatric trainees are proposed to play a leading role in this new subspeciality of Paediatrics, gaining valuable clinical and research experience on the prevention and management of viral infections in neonates and children (Fig. 1). They will be involved in the clinical care for hospitalised children with uncommon, recurrent, severe or complex neonatal and paedi- 


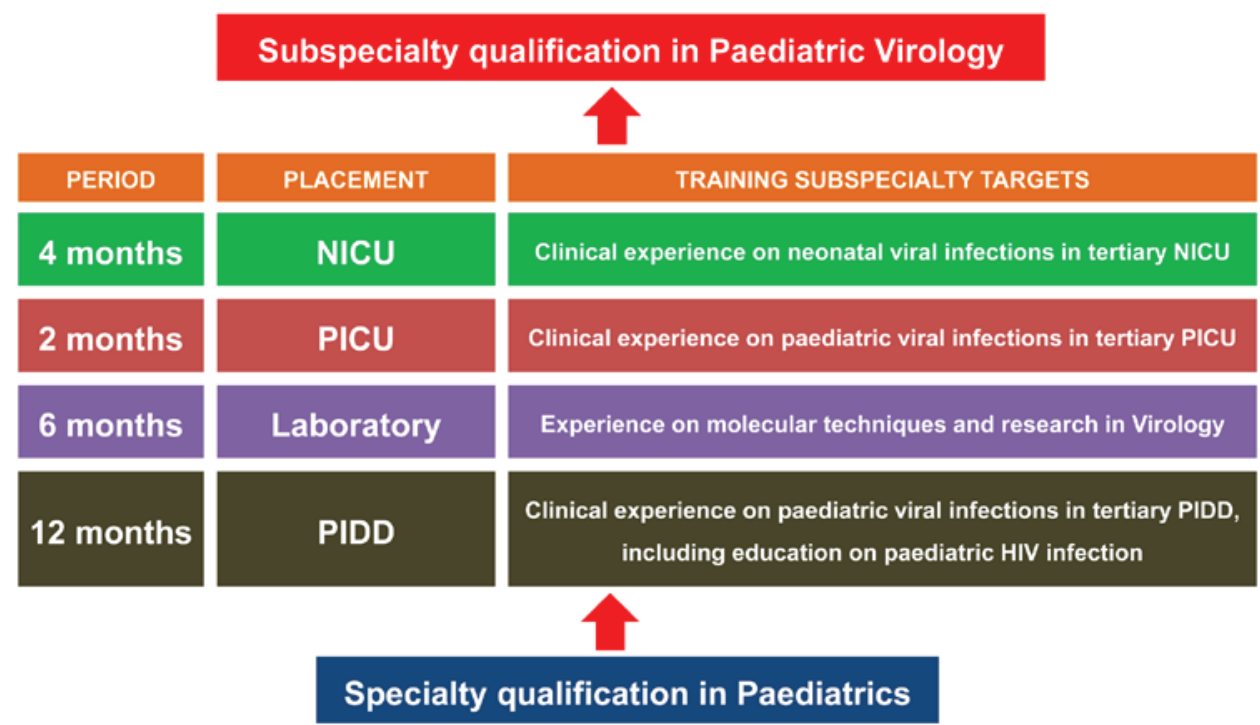

Figure 1. The proposed subspecialty training programme of Paediatric Virology. HIV, human immunodeficiency virus; NICU, Neonatal Intensive Care Unit; PICU, Paediatric Intensive Care Unit; PIDD, Paediatric Infectious Diseases Department.

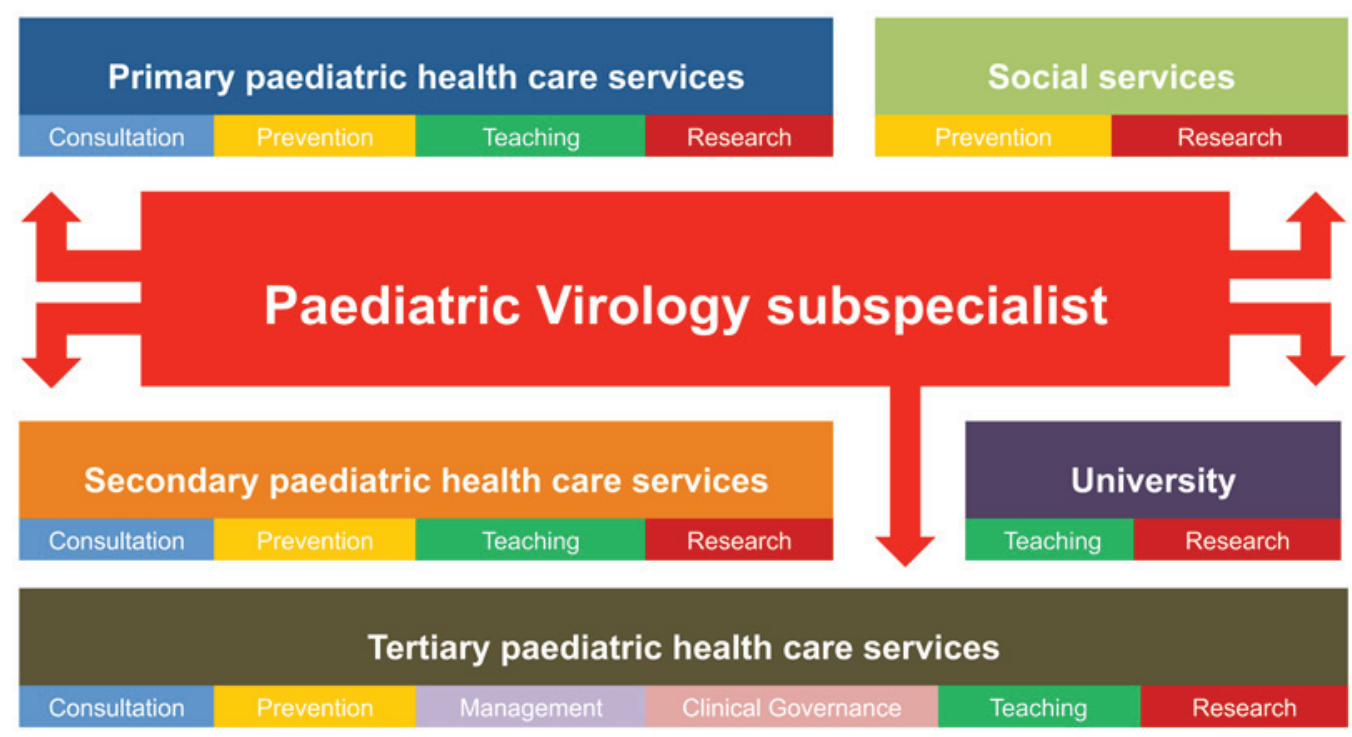

Figure 2. The proposed multi-task role of Paediatric Virology subspecialists in primary, secondary and tertiary paediatric services, university-based research and educational settings.

atric viral infections and will focus on their comprehensive assessment and treatment. Moreover, they will enhance the coordination of hospital and community care teams and offer appropriate consultation and assistance. After their qualification, Paediatric Virology subspecialists will play a multi-task role, not only in university-based research and educational settings, but also in primary, secondary and tertiary paediatric services (Fig. 2). Their increased accessibility can potentially result in cost savings to the health care system and better quality of care for paediatric patients. This will be achieved by limiting the unnecessary utilisation of emergency rooms, the multiple clinical visits to various health care providers and the request of unwarranted investigations.

It is important to determine whether and how Paediatric Virology as a new paediatric subspecialty can help paediatric clinical practice. In the present era of job limitations due to the financial crisis, it would be interesting to determine whether this subspecialty can support educational and academic strategies promoting current paediatric health and prevention. Without any doubt, our proposal requires further evaluation and discussion. The official recognition of Paediatric Virology by the key worldwide scientific and academic paediatric stakeholders will allow the development of an accredited training programme able to attract the highest quality paediatric trainees. In the future, Paediatric Virology subspecialists will be expected to have a strategically principal role, both clinical and academic, at the fight against viral infections in childhood. These efforts will aim to offer a state-of-the-art continuous medical education provided by both clinicians and basic scientists. This definitely needs more time, passion and inspiration. 


\section{References}

1. Stoddard JJ, Cull WL, Jewett EA, Brotherton SE, Mulvey HJ and Alden ER: AAP Providing pediatric subspecialty care: A workforce analysis. AAP Committee on Pediatric Workforce Subcommittee on Subspecialty Workforce. Pediatrics 106: 1325-1333, 2000.

2. Noel GJ, Stavola JJ and Schauf V: Paediatric infectious diseases: A comprehensive guide to the subspecialty. Johns Hopkins University Press, Baltimore, MD, 1997.

3. Starr M: Paediatric infectious diseases: The last 50 years. J Paediatr Child Health 51: 12-15, 2015.

4. Shulman ST: The history of pediatric infectious diseases. Pediatr Res 55: 163-176, 2004.
5. Danziger-Isakov L, Evans HM, Green M, McCulloch M, Michaels MG, Posfay-Barbe KM, Verma A and Allen U; ID CARE Committee from IPTA: Capacity building in pediatric transplant infectious diseases: an international perspective. Pediatr Transplant 18: 790-793, 2014.

6. Rubin LG, Levin MJ, Ljungman P, Davies EG, Avery R, Tomblyn M, Bousvaros A, Dhanireddy S, Sung L, Keyserling $\mathrm{H}$, et al; Infectious Diseases Society of America: 2013 IDSA clinical practice guideline for vaccination of the immunocompromised host. Clin Infect Dis 58: 309-318, 2014.

7. Barker CI, Germovsek E, Hoare RL, Lestner JM, Lewis J and Standing JF: Pharmacokinetic/pharmacodynamic modelling approaches in paediatric infectious diseases and immunology. Adv Drug Deliv Rev 73: 127-139, 2014. 